\title{
Unusual Masses of the Pancreas to Be Aware of
}

\author{
Muriel Genevay ${ }^{\mathrm{a}} \quad$ Jean Marc Dumonceau ${ }^{\mathrm{b}} \quad$ Thierry Berney $^{\mathrm{c}}$

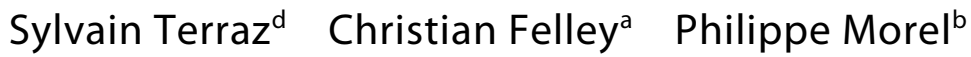 \\ Jean Louis Frossard ${ }^{\mathrm{b}}$ \\ aDivision of Clinical Pathology, bService of Gastroenterology and Hepatology, \\ 'Service of Digestive Surgery, and dDepartment of Radiology, Geneva University \\ Hospitals, Geneva, Switzerland
}

\section{Key Words}

Unusual masses · Pancreas · Ductal pancreatic adenocarcinoma

\begin{abstract}
This paper aims at emphasizing the difficulty in assessing preoperatively the diagnosis of solid masses of the pancreas whatever the initial clinical presentation may be. We illustrate our purpose describing consecutive cases of pancreatic masses of the pancreas we recently had and who were followed according to the internal guidelines of investigation of our referral hospital. Whereas malignant tumors of the pancreas represent the vast majority of solid tumors of the pancreas, other diagnoses must be evoked. We report three cases of pancreatic solid masses that were explored by endoscopic ultrasonography coupled with fine needle aspiration, a method universally considered to be both reliable and accurate but which failed to assess definitive diagnosis due to both cytological pitfalls and sampling error.
\end{abstract}

\section{Introduction}

Ductal pancreatic adenocarcinoma is by far the first diagnosis to evoke when dealing with a solid mass of the pancreas as its frequency reaches 85-95\% [1]. However, other diagnoses must be considered such as endocrine tumors which may account for $1-3 \%$ of pancreatic tumors, solid and pseudopapillary tumor or benign pseudotumoral lesions such as autoimmune pancreatitis $[2,3]$. Even rarer are metastatic tumors to the pancreas or mesenchymal and hematopoietic cancers arising in the pancreas (liposarcoma, leiomyosarcoma, fibrosarcoma, and lymphoma) [4]. These differential diagnoses depend on clinical presentation: solid and pseudopapillary tumors, for example, although rare, are frequently evoked in women of child-bearing age [5]. 
To avoid surgery when not indicated, endoscopic ultrasonography (EUS) coupled with fine needle aspiration (FNA) has been universally recognized as efficient and safe [6]. Indeed, EUS-FNA is feasible in $90-95 \%$ of all pancreatic masses and its performance in collecting adequate tissue to get a final diagnosis is close to $80-95 \%$ [6-8]. However, sampling or interpretation errors can lead to misdiagnosis.

We present three cases where clinical presentation, sampling error or cytologic interpretation led to a wrong preoperative diagnosis.

\section{Case 1}

A 17-year-old otherwise healthy woman presented with a 2-month history of recurrent epigastric pain without nausea nor vomiting. Physical examination was unremarkable. C-reactive protein was within normal range. Her liver tests were normal whereas serum lipase was increased at $609 \mathrm{U} / \mathrm{l}$ (normal 114-329). Serum IgG4 was within normal range. Abdominal ultrasound was normal. Computed tomography scan showed a $4 \times 2 \mathrm{~cm}$ hypodense mass of the pancreatic tail only seen during the arterial phase of the scan (fig. 1a). She was referred to us for EUS-FNA. EUS demonstrated a hypoechoic heterogeneous mass of the pancreatic tail (fig. 1b). FNA brought back a very scant material consisting of few monotonous oval cells with round to oval nuclei and moderate cytoplasm. In addition, hyaline globules were present and led the cytopathologist, in this particular clinical setting, to assess the diagnosis of solid and pseudopapillary tumor of the pancreas, previously referred to as papillary cystic tumor and papillary cystic neoplasm. The patient was then referred to the surgeon for a left pancreatectomy. A partial pancreatectomy was realized. On final macroscopic examination, the pancreas appeared diffusely enlarged, without any visible mass. On histological examination, pancreatic parenchyma was modified by abundant fibrosis accompanied by a florid inflammatory infiltrate. This infiltrate set predominantly around the pancreatic ducts and was mainly composed of plasmocytes. The final histological diagnosis was autoimmune pancreatitis. The postoperative period was characterized by recurrent abdominal pain and persistence of increased lipase. Steroids were then introduced for 2 months with a complete absence of pain and normalization of serum lipase.

\section{Case 2}

A 58-year-old woman without any relevant past medical history presented with a 2-month period of recurrent abdominal pain associated with significant weight loss of $6 \mathrm{~kg}$. There was no history of tobacco or alcohol use. Laboratory investigation was unremarkable. Serum IgG4 was within normal range. CT scan showed a large hypodense mass of the pancreatic body, $50 \mathrm{~mm}$ in size (fig. $2 \mathrm{a}$ ). EUS demonstrated a large $45 \mathrm{~mm}$ hypoechoic mass of the uncinatus process, very well delineated with a hyperechoic wall (fig. 2b). PET-CT scan identified a large hypermetabolic spot within the head of the pancreas corresponding to the tumor seen at CT (fig. 2c). EUS-FNA was performed, but it was very poorly cellular and was mainly composed of gastrointestinal cells and thus not conclusive. The patient was sent to the surgeon with the putative diagnosis of solid and pseudopapillary tumor of the pancreas based on her history and imaging studies. A duodenopancreatectomy was performed. A $3.5 \mathrm{~cm}$ mass was present in the head of the pancreas, mainly solid but with a central necroticohemorragic aspect. This tumor was delineated by a dense fibrous capsule. The final histological diagnosis was pancreatoblastoma due to the presence of both endocrine and acinar cells associated with squamoid nest. The immunohistochemical study showed a predominant endocrine component and a peculiar staining for $\beta$-catenin, centred on squamoid nests as it has been previously described [9]. Seven lymph nodes were retrieved, all negative. No adjuvant treatment was initiated.

\section{Case 3}

A 67-year-old man with a past history of alcohol abuse presented with a first attack of acute pancreatitis characterized by abdominal pain associated with a serum lipase elevated at $785 \mathrm{U} / \mathrm{l}$. The patient was a heavy smoker. Physical examination upon admission revealed moderate epigastric tenderness that disappeared within $6 \mathrm{~h}$ after admission. CRP was within normal range. His liver tests were normal. CT scan showed a normal gallbladder without stone and a focal mass of $2 \mathrm{~cm}$ in the tail of the pancreas (fig. $3 a)$. EUS identified irregular main duct and side branch duct with hyperechoic and 
thickened wall, pseudolobular aspect of the pancreatic parenchyma with hyperechoic strands and a solid hyperechoic mass of $25 \mathrm{~mm}$ in size located in the tail of the pancreas (fig. 3b) and in close contact with the splenic vein. Hormones including insulin, glucagon, vasointestinal peptide, chromogranin and serotonin were within normal range. Octreoscan identified a hyperactive spot 5 and $24 \mathrm{~h}$ after tracer injection in the tail of the pancreas highly suggestive of a neuroendocrine tumor (fig. 3c). EUS-FNA was performed and retrieved hemorrhagic material with scattered lymphocytes and was thus considered as inconclusive. Left pancreatectomy with spleen preservation was performed. A $2 \mathrm{~cm}$ well-delineated brown mass similar to spleen was present within the pancreatic parenchyma. Histology confirmed the splenic nature of the lesion and then assessed a diagnosis of intrapancreatic accessory spleen.

\section{Discussion}

There are many different types of tumors that can develop in the pancreas. Approximately 85-90\% of solid masses are adenocarcinomas of the pancreas [10]. The remaining $10-15 \%$ correspond, most of the time, to less aggressive types of tumors which are often curable [11]. We report three cases of pancreatic solid masses that were initially explored by CT scan and then by EUS-FNA, a method universally considered to be both reliable and accurate [12]. In our cases, however, EUS-FNA failed to assess a definitive diagnosis due to both cytological pitfalls and sampling error.

In the first case, EUS-FNA brought relatively bland-looking cells with peculiar hyaline globules, which in retrospect corresponded either to ductular or gastrointestinal cells as has been previously reported [13-15]. The hyaline globules were probably due to epithelial degeneration. Smears lacked the cellular inflammatory stromal fragments highly suggestive of autoimmune pancreatitis and were also completely devoid of inflammatory cells [13]. However, low sensitivity of EUS-FNA in a context of autoimmune pancreatitis is well described [13-15]. EUS studies have shown that EUS-trucut biopsy could serve as a rescue technique in cases of autoimmune pancreatitis lacking typical findings [16], a feature already reported for EUS-FNA [17] but associated with a higher rate of complications.

In the second case, EUS-FNA was unable to bring enough material. For definitive diagnosis, expert consultation was required [18]. Pancreatoblastomas are indeed rare in adults, and this one presented a peculiar morphological aspect. The endocrine component was heavily predominant and the squamoid nests were barely visible. That is why the diagnosis of endocrine tumor was first evoked. However, immunostaining for $\beta$-catenin was typical with an intense nuclear staining in squamoid nests and a membranous staining in endocrine and acinar component. Even though $\beta$-catenin has been rarely described as having nuclear staining in pancreatic endocrine tumors, it presents a heterogeneous distribution very different from the systematized staining obtained here [19].

In the third case, the clinical and biological data suggesting a nonfunctional endocrine tumor were strongly supported by the octreoscan results. EUS-FNA retrieved a lot of red cells coupled to lymphocytes and no other cell type, a feature that has to be confronted to the final diagnosis of intrapancreatic accessory spleen, a rare benign condition [20]. A small radiological series showed that magnetic resonance imaging may be the diagnostic test to use in such a suspicion [21]. Indeed, the discovery of a well-marginated, rounded mass in the distal aspect of the tail of the pancreas with signal intensity features of the spleen on all precontrast and postgadolinium sequences suggests the diagnosis of intrapancreatic accessory spleen [21]. 


\begin{tabular}{r|l|l|l} 
Case Reports $h$ & $\begin{array}{l}\text { Case Rep Gastroenterol 2009;3:389-394 } \\
\text { D01: 10.1159/000255401 }\end{array}$ & Published online: November 21, 2009 & $\begin{array}{l}\text { O 2009 S. Karger AG, Basel } \\
\text { ISSN 1662-0631 } \\
\text { www.karger.com/crg }\end{array}$ \\
\hline
\end{tabular}

In these three cases, EUS-FNA failed to make the right preoperative diagnosis for different reasons. In the first case, the clinical presentation (a tumor located in the pancreatic tail in a young woman) forced the pathologist to overinterpret contaminant as tumor cells. The final diagnosis of autoimmune pancreatitis was however quite surprising in such a clinical context as it is described to develop around the age of 50. In the second case, the clinical suspicion of solid and pseudopapillary tumor was high enough to undertake surgery despite the inconclusive cytological examination. Here again, the diagnosis of pancreatoblastoma in an adult was so rare that it was not even evoked preoperatively. In the third case, the lesion was indeed correctly sampled as FNA brought both blood and lymphocytes coming from the spleen. However, as this diagnosis was not even suspected clinically and radiologically, this was not even taken into consideration.

EUS-FNA is known to need clinical correlation to be interpreted, but the initial clinical presentation may bias the cytological or imaging results. These three observations emphasize the importance of considering other diagnoses before sending the patient to the surgeon for a procedure that carries a substantial risk of complications and mortality.

Fig. 1. a CT scan showing a $4 \times 2 \mathrm{~cm}$ hypodense mass of the pancreatic tail. b EUS showing a heterogenous hypoechoic mass of the tail of the pancreas using a sectorial endoscope at the time of FNA.
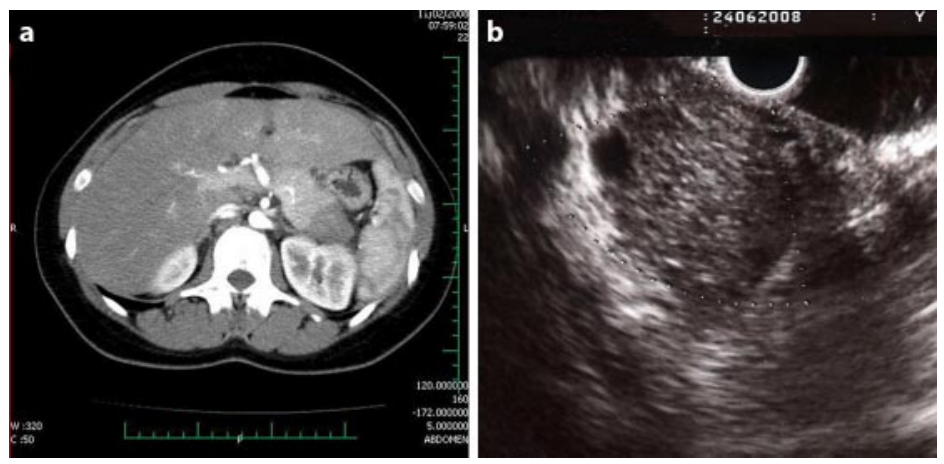

Fig. 2. a CT scan with a large corporeal hypodense mass of the pancreas with fine delineated hyperechoic wall. b The mass seen at EUS was hypoechoic with a strong heterogenous content. c PET-CT demonstrated a huge hypermetabolic state of the pancreatic mass.
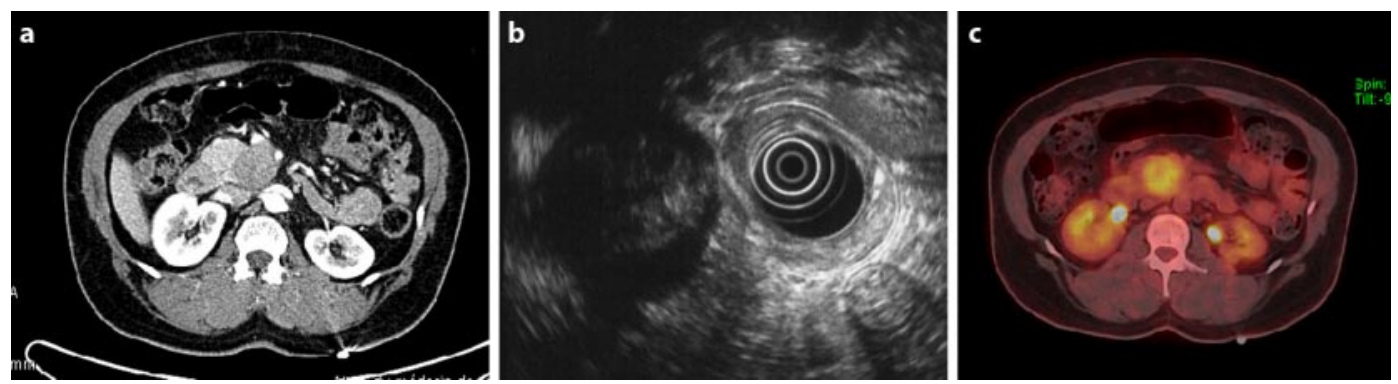
Fig. 3. a CT showed a well-delineated mass of the pancreatic tail without upstream ductular dilatation. b A small homogenous mass of the pancreas was demonstrated by EUS at the time of FNA. Note that the mass was very well delineated. c Octreoscan identified a hyperintense spot located in the tail of the pancreas when compared to the spleen.
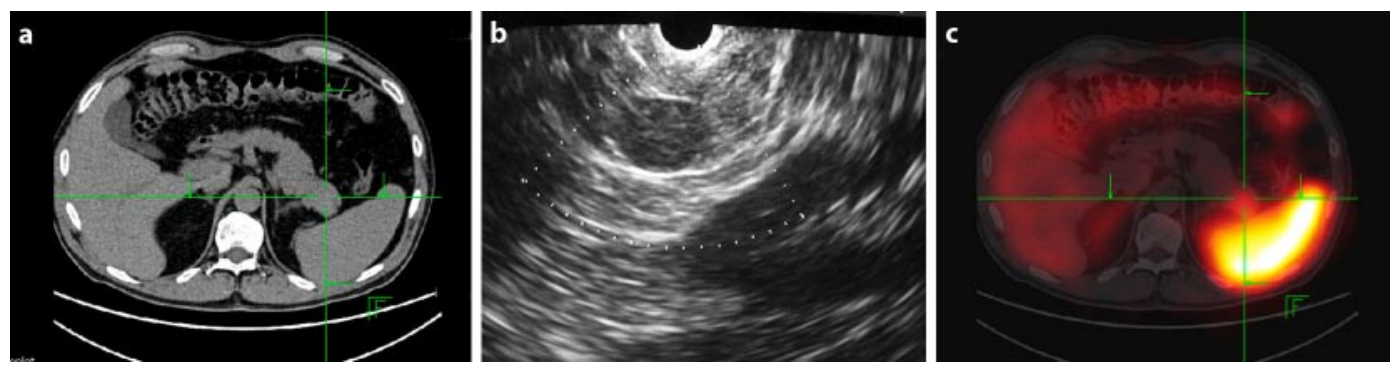


\section{References}

1 Klimstra D, Pitman M, Hruban R: An algorithmic approach to the diagnosis of pancreatic neoplasms. Arch Pathol Lab Med 2009;133:454-464.

2 de Suray N, Van Beers B, Sempoux C, et al: Autoimmune pancreatitis, sclerosing cholangitis, liver inflammatory pseudotumors, retroperitoneal fibrosis, lymphadenopathy, and sialoadenitis in a single patient: a multifaceted expression of a hitherto unrecognized systemic autoimmune syndrome? Pancreas 2009;38:476-478.

3 Frossard JL, Steer M, Pastor C: Acute pancreatitis. Lancet 2008;371:143-152.

4 Levey J, Banner B: Adult pancreatoblastoma: a case report and review of the literature. Am J Gastroenterol 1996;91:1841-1844.

5 Garcea G, Ong S, Rajesh A, et al: Cystic lesions of the pancreas. A diagnostic and management dilemma. Pancreatology 2008;8:236-251.

6 Wiersema M, Vilmann P, Giovannini M, Chang K, Wiersema L: Endosonography-guided fine-needle aspiration biopsy: diagnostic accuracy and complication assessment. Gastroenterology 1997;112:1087-1095.

7 Scheiman J: Management of cystic lesions of the pancreas. J Gastrointest Surg 2008; 12:405-407.

8 tenBerge J, Hoffman B, Hawes R, et al: EUS-guided fine needle aspiration of the liver: indications, yield, and safety based on an international survey of 167 cases. Gastrointest Endosc 2002;55:859-862.

9 Tanaka Y, Kato K, Notohara K, et al: Significance of aberrant (cytoplasmic/nuclear) expression of beta-catenin in pancreatoblastoma. J Pathol 2003;199:185-190.

10 Jemal A, Siegel R, Ward E, et al: Cancer statistics, 2006. CA Cancer J Clin 2006;56:106-130.

11 Reddy S, Wolfgang C: Benign pancreatic tumors. Surg Clin North Am 2007;87:1359-1378.

12 Frossard JL, Amouyal P, Amouyal G, et al: Performance of endosonography-guided fine needle aspiration and biopsy in the diagnosis of pancreatic cystic lesions. Am J Gastroenterol 2003;98:1516-1524.

13 Deshpande V, Mino-Kenudson M, Brugge W, et al: Endoscopic ultrasound guided fine needle aspiration biopsy of autoimmune pancreatitis: diagnostic criteria and pitfalls. Am J Surg Pathol 2005;29:1464-1471.

14 Fu K, Eloubeidi MA, Jhala NC, Jhala D, Chhieng DC, Eltoum IE: Diagnosis of gastrointestinal stromal tumor by endoscopic ultrasound-guided fine needle aspiration biopsy - a potential pitfall. Ann Diagn Pathol 2002;6:294-301.

15 Wang B, Hunter W, Bin-Sagheer S, Bewtra C: Rare potential pitfall in endoscopic ultrasound-guided fine needle aspiration biopsy in gastric duplication cyst: a case report. Acta Cytol 2009;53:219-222.

16 Mizuno N, Bhatia V, Hosoda W, et al: Histological diagnosis of autoimmune pancreatitis using EUS-guided trucut biopsy: a comparison study with EUS-FNA. J Gastroenterol 2009;12:110-114.

17 Salla C, Chatzipantelis P, Konstantinou P, Karoumpalis I, Pantazopoulou A, Tsiotos G: EUS-FNA contribution in the identification of autoimmune pancreatitis: a case report. JOP 2007;8:598-604.

18 Savastano S, d'Amore E, Zuccarotto D, Banzato O, Beghetto M, Famengo B: Pancreatoblastoma in an adult patient. A case report. JOP 2009;10:192-195.

19 Hervieu V, Lepinasse F, Gouysse G, et al: Expression of beta-catenin in gastroenteropancreatic endocrine tumours: a study of 229 cases. J Clin Pathol 2006;59:1300-1304.

20 Kim S, Lee J, Han J, et al: Intrapancreatic accessory spleen: findings on MR Imaging, CT, US and scintigraphy, and the pathologic analysis. Korean J Radiol 2008;9:162-174.

21 Herédia V, Altun E, Bilaj F, et al: Gadolinium- and superparamagnetic-ironoxide-enhanced MR findings of intrapancreatic accessory spleen in five patients. Magn Reson Imaging 2008;26:1273-1278. 